\title{
Aspectos metodológicos do comportamento ingestivo de ovinos alimentados com capim-elefante amonizado e subprodutos agroindustriais ${ }^{1}$
}

\author{
Gleidson Giordano Pinto de Carvalho², Aureliano José Vieira Pires ${ }^{3,7}$, Robério Rodrigues \\ Silva ${ }^{4}$, Bruna Mara Aparecida de Carvalho ${ }^{5}$, Herymá Giovane de Oliveira Silva ${ }^{3}$, Lorendane \\ Millena de Carvalho 6
}

\footnotetext{
${ }^{1}$ Projeto financiado pela Universidade Estadual do Sudoeste da Bahia - UESB, Itapetinga, BA.

2 Doutorando em Zootecnia, UFV, Viçosa, MG. Bolsista do CNPq.

${ }^{3}$ Departamento de Tecnologia Rural e Animal, UESB, Itapetinga, BA.

${ }^{4}$ Departamento de Estudos Básicos e Instrumentais, UESB, Itapetinga, BA. Doutorando em Zootecnia, UEM, Maringá, PR.

5 Mestranda em Ciência e Tecnologia de Alimentos, UFV, Viçosa, MG.

${ }^{6}$ Graduanda em Medicina Veterinária, UFES, Alegre, ES.

7 Pesquisador do CNPq.
}

RESUMO - Um experimento foi realizado com o objetivo de definir o intervalo de tempo de registro do comportamento de ovinos Santa Inês. Utilizaram-se 18 ovinos machos, não-castrados, com peso corporal médio inicial de 22,62 kg. Os animais foram alojados em baias individuais e distribuídos ao acaso em seis tratamentos, constituídos de dois volumosos (capim-elefante amonizado ou não com $5 \%$ de uréia) e três concentrados (com $0 \%$ de farelo de cacau ou torta de dendê; com $40 \%$ de farelo de cacau; ou com 40\% de torta de dendê em substituição ao milho e ao farelo de soja), fornecidos na proporção de 60:40 (volumoso:concentrado). O registro das atividades foi realizado durante dois períodos de 24 horas, no final do período experimental, registrando-se o tempo despendido em alimentação, ruminação, ócio e efetuando-se a discretização dessas séries. Foram testados os intervalos de observações de 5, 10, 15, 20, 25 e 30 minutos. Os tempos despendidos em alimentação, ruminação e ócio não diferiram em nenhum dos intervalos estudados, entretanto, na discretização das séries temporais, ou seja, no número de períodos e no tempo médio gasto por período, apresentaram diferenças significativas. Os tempos de alimentação, ruminação e ócio em ovinos Santa Inês podem ser obtidos com observações em intervalos de até 30 minutos. Para a discretização das séries temporais, recomenda-se o uso da escala de cinco minutos.

Palavras-chave: amonização, comportamento alimentar, escala, farelo de cacau

\section{Methodological aspects of feeding behaviour of sheep fed ammoniated elephantgrass and agro-industrial by-products}

\begin{abstract}
The objective of this trial was to compare and determine the most adequate time interval to register chewing activity in Santa Inês sheep. The following time intervals were investigated: 5, 10, 15, 20, 25, and 30 minutes. Eighteen intact male sheep averaging $22.62 \mathrm{~kg}$ of body weight at the beginning of the study were used. Animals were housed in individual pens and randomly assigned to one of six treatments: ammonia-treated elephantgrass or elephatgrass supplemented with three concentrates sources ( $0 \%$ cocoa meal and palm kernel cake, $40 \%$ cocoa meal, and $40 \%$ palm kerne cake partially replacing corn and soybean meal) in a forage:concentrate ratio of 60:40. Chewing activity (time spent eating, ruminanting, and idle) was measured during two 24 hours periods at the end of the experimental period. There was no significant difference in the time spent eating, ruminating and idle among the studied intervals. However, significant differences were observed for the number of meals, rumination, and idle activities as well as for the mean time spent at each of these individual activities. It can be concluded that time spent eating, ruminating, and idle can be measured at every 30 minutes interval in Santa Inês sheep while five minutes interval was required to register the number and the mean time spent at each chewing activity.
\end{abstract}

Key Words: ammoniation, cocoa meal, feeding behaviour, scale

\section{Introdução}

O desempenho animal depende essencialmente da nutrição, no entanto, a despreocupação com o comporta- mento de ovinos tem ocasionado limitações no entendimento de algumas respostas encontradas nas pesquisas.

$\mathrm{O}$ estudo do comportamento ingestivo pode elucidar problemas relacionados à diminuição do consumo em épocas 
críticas, atribuída aos efeitos das práticas de manejo e dimensionamento das instalações, da qualidade e da quantidade da dieta (Albright, 1993).

Com os recentes avanços na área de etologia, a escolha de um adequado intervalo de tempo para o registro do comportamento ingestivo que permita a observação de maior número de animais e que não se contraponha à avaliação precisa dos aspectos comportamentais tem sido amplamente discutida e estudada por pesquisadores em todo o País.

O método de observação contínuo, sem intervalos, foi avaliado simultaneamente com as escalas de observação de 5, 7, 10 e 15 minutos por Salla et al. (1999), utilizando vacas Jersey. Recentemente, Carvalho et al. (2007) testaram as escalas de 10, 15 e 20 minutos em comparação ao intervalo de cinco minutos em experimento com cabras leiteiras da raça Saanen e Silva et al. (2004) avaliaram escalas de até 30 minutos em pesquisa com novilhas 3/4 Holandês $\times$ Zebu.

A necessidade de intensificação dos sistemas de produção de carne ovina no Brasil tem motivado pesquisadores e técnicos a buscarem alternativas que possibilitem melhores combinações de alimentos. No entanto, esperam-se modificações no comportamento ingestivo dos animais, pois, segundo Campbell et al. (1992), os fatores que afetam o comportamento ingestivo estão relacionados ao alimento, ao ambiente e ao animal.

A aplicação de uréia em volumosos com o intuito de melhorar o valor nutritivo tem sido muito empregada. Os principais benefícios do tratamento desses volumosos são o aumento do teor de PB pela adição de nitrogênio nãoprotéico e a redução do teor de fibra a partir da solubilização parcial da hemicelulose (Pires et al., 2003). Outra técnica de alimentação muito utilizada é a adição de resíduos agroindustriais nas dietas, especialmente de ruminantes. $\mathrm{O}$ uso de resíduos é fundamental, principalmente quando o objetivo é reduzir o custo de produção. O farelo de cacau, resíduo obtido no processo de torrefação da amêndoa do cacau, após obtenção da manteiga e do chocolate, assim como a torta de dendê, obtida após a extração do óleo, podem ser empregados na alimentação de ruminantes (Carvalho et al., 2004; Silva et al., 2005a), no entanto, seus efeitos no comportamento animal ainda são desconhecidos.

Os padrões de comportamento constituem-se um dos meios mais efetivos pelos quais os animais adaptam-se a diversos fatores ambientais, portanto, podem indicar métodos potenciais de melhoramento da produtividade animal com a utilização de diferentes manejos. Entretanto, a correta compreensão de um fenômeno depende do estudo da metodologia de avaliação (Salla et al., 1999).

A escolha do intervalo para discretizar as séries temporais, ou seja, o número e o tempo médio gasto por períodos de alimentação, ruminação e descanso, deve ser uma ponderação entre o poder de detectar mudanças na ocorrência das atividades e a precisão, sem, no entanto, incorrer em redundância. O intervalo de cinco minutos entre observações tem sido adotado como padrão na maioria das pesquisas (Moore et al., 1990; Bürger et al., 2000; Queiroz et al., 2001; Salla et al., 2003; Mendonça e al., 2004), por ser o que mais se aproxima do método de observação contínua. Deswysen et al. (1993) utilizaram o intervalo de sete minutos, enquanto outros autores utilizaram o intervalo de 10 minutos (Miranda et al., 1999; Gonçalves et al., 2001; Costa et al., 2003; Carvalho et al., 2004) ou de 15 minutos (Fischer et al., 1998; Portugal et al., 2000; Silva et al., 2005b). Todavia, na maioria dos trabalhos, a escolha da escala foi realizada de forma totalmente aleatória e, por afetar a percepção do observador quanto à heterogeneidade do sistema, sua adoção inadequada pode comprometer os resultados (Dutilleul, 1997).

Este trabalho foi realizado com o objetivo de definir o intervalo de registro do comportamento de ovinos Santa Inês alimentados com capim-elefante (Pennisetum purpureum, Schum) amonizado ou não com uréia (5\% na base da MS) e farelo de cacau (Theobroma cacao L.) ou torta de dendê (Elaeis guineesis, Jacq) como parte da dieta.

\section{Material e Métodos}

O experimento foi realizado nas dependências do Setor de Ovinocultura da Universidade Estadual do Sudoeste da Bahia no período de abril a setembro de 2003.

Foram utilizados 18 ovinos Santa Inês com peso médio inicial de 22,62 kg, alojados em baias individuais com 1,2 $\mathrm{m}^{2}$ de área e piso ripado de madeira, providas de comedouros e bebedouros, dispostos frontalmente em cada baia.

As dietas oferecidas foram compostas de volumoso e concentrado, na proporção de 60:40. Os concentrados foram formulados para serem isoprotéicos (16\% de PB). Os animais foram distribuídos em seis tratamentos (dietas experimentais), compostos de capim-elefante (Pennisetum purpureum, Schum) amonizado ou não com uréia (5,0\% na MS) e três concentrados: controle - milho + farelo de soja; controle com $40 \%$ de farelo de cacau (Theobroma cacao L.); e controle com $40 \%$ de torta de dendê (Elaeis guineesis, Jacq) (Tabela 1).

Os alimentos foram fornecidos duas vezes ao dia, às $8 \mathrm{~h}$ e às $15 \mathrm{~h} 30$, em quantidade ajustada para proporcionar $10 \%$ de sobras nos cochos, com consumo de água ad libitum.

A composição química dos volumosos, dos concentrados e das dietas experimentais é descrita nas Tabelas 2 e 3 . Todas as análises de alimentos foram realizadas segundo procedimentos descritos por Silva \& Queiroz (2002). 
O experimento teve duração de 78 dias. Os primeiros 15 dias foram destinados à adaptação dos animais às dietas e ao ambiente e os dois últimos, à determinação do comportamento alimentar nas diferentes escalas $(5,10,15,20,25$ e 30 minutos), pela quantificação dos intervalos de cada

Tabela 1 - Proporção dos ingredientes (\%) no concentrado, com base na MS

Table 1 - Ingredient composition of concentrate, on DM basis

\begin{tabular}{lccc}
\hline $\begin{array}{l}\text { Ingrediente } \\
\text { Ingredient }\end{array}$ & \multicolumn{3}{c}{$\begin{array}{c}\text { Concentrado } \\
\text { Concentrate }\end{array}$} \\
\cline { 2 - 4 } & Controle & $\begin{array}{c}\text { Controle }+40 \% \\
\text { farelo de cacau } \\
\text { Control plus } \\
\text { 40\% cocoa meal }\end{array}$ & $\begin{array}{c}\text { Controle }+40 \% \\
\text { torta dendê } \\
\text { Control plus } \\
40 \% \text { palm cake }\end{array}$ \\
\hline $\begin{array}{l}\text { Milho moído } \\
\text { Ground corn }\end{array}$ & 76,85 & 41,27 & 43,97 \\
$\begin{array}{l}\text { Farelo de soja } \\
\text { Soybean meal }\end{array}$ & 20,19 & 16,17 & 13,47 \\
$\begin{array}{l}\text { Farelo de cacau } \\
\text { Cocoa meal }\end{array}$ & 0,00 & 39,59 & 0,00 \\
$\begin{array}{l}\text { Torta de dendê } \\
\text { Palm cake }\end{array}$ & 0,00 & 0,00 & 39,57 \\
$\begin{array}{l}\text { Mistura mineral } \\
\text { Mineral mixture }\end{array}$ & 1,97 & 1,99 & 1,99 \\
$\begin{array}{l}\text { Sal comum } \\
\text { Salt }\end{array}$ & 0,99 & 0,99 & 0,99 \\
\hline
\end{tabular}

atividade, durante 24 horas por dia, para cada animal. A duração média diária dos períodos das atividades foi calculada dividindo-se a duração total de cada atividade pelo seu respectivo número de períodos discretos.

O registro do tempo despendido em alimentação, ruminação ou ócio foi realizado por dois observadores treinados, em sistema de revezamento, posicionados estrategicamente de forma a não incomodar os animais. Durante a observação noturna, o ambiente foi mantido com iluminação artificial.

Como tratamentos foram considerados os dois volumosos, os três concentrados e as seis escalas, com seis repetições, ou seja, três animais por tratamento $\times$ dois dias de observação. As análises estatísticas dos dados foram realizadas utilizando-se o programa SAEG - Sistema de Análises Estatísticas e Genéticas (UFV, 2000) e os resultados foram interpretados estatisticamente por meio de análise de variância e do teste Tukey, a 5\% de probabilidade, segundo o modelo estatístico:

$\mathrm{Y}_{\mathrm{ijlr}}=\mu+\mathrm{N}_{\mathrm{i}}+\mathrm{P}_{\mathrm{j}}+\mathrm{Q}_{1}+\mathrm{NP}_{\mathrm{ij}}+\mathrm{NQ}_{\mathrm{il}}+\mathrm{PQ}_{\mathrm{jl}}+\mathrm{NPQ}_{\mathrm{ijl}}+\mathrm{e}_{\mathrm{ijl} \mathrm{r}}$ em que: $Y_{i j l r}=$ observação referente ao animal r na fonte de volumoso $\mathrm{i}$, do concentrado $\mathrm{j}$ e da escala $\mathrm{l} ; \mu$ = média geral; $\mathrm{N}_{\mathrm{i}}=$ efeito da fonte de volumoso $\mathrm{i}(\mathrm{i}=1,2) ; \mathrm{P}_{\mathrm{j}}=$ efeito do

Tabela 2 - Composição química dos volumosos e do concentrado (\% MS)

Table 2 - Chemical composition of forages and concentrate (\% MS)

\begin{tabular}{|c|c|c|c|c|c|}
\hline \multirow[t]{2}{*}{$\begin{array}{l}\text { Nutriente } \\
\text { Item }\end{array}$} & \multicolumn{2}{|c|}{$\begin{array}{l}\text { Volumoso } \\
\text { Forage }\end{array}$} & \multicolumn{3}{|c|}{$\begin{array}{l}\text { Concentrado } \\
\text { Concentrate }\end{array}$} \\
\hline & $\begin{array}{l}\text { Não amonizado } \\
\text { Not ammoniated }\end{array}$ & $\begin{array}{l}\text { Amonizado } \\
\text { Ammoniated }\end{array}$ & $\begin{array}{l}\text { Controle } \\
\text { Control }\end{array}$ & $\begin{array}{c}40 \% \text { farelo de cacau } \\
40 \% \text { cocoa meal }\end{array}$ & $\begin{array}{c}40 \% \text { torta de dendê } \\
40 \% \text { palm cake }\end{array}$ \\
\hline $\mathrm{PB}(C P)$ & 4,13 & 7,15 & 16,87 & 16,91 & 16,41 \\
\hline FDN (NDF) & 70,31 & 69,63 & 12,37 & 25,47 & 39,16 \\
\hline FDA $(A D F)$ & 34,47 & 33,07 & 4,44 & 18,27 & 21,09 \\
\hline NIDA (ADIN) & 0,19 & 0,23 & 0,25 & 0,26 & 0,26 \\
\hline Cinzas (Ash) & 11,25 & 10,96 & 9,14 & 8,67 & 8,91 \\
\hline
\end{tabular}

Tabela 3 - Composição química das dietas experimentais (\% da MS)

Table 3 - Chemical composition of the experimental diets (\% of DM)

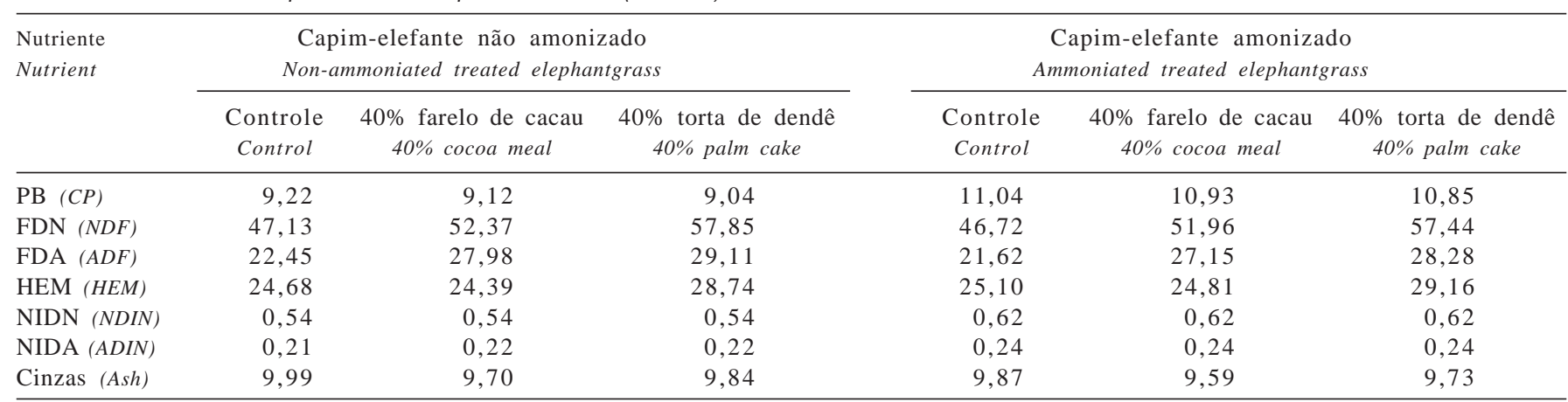


concentrado j $(\mathrm{j}=1,2,3) ; \mathrm{Q}_{1}=$ efeito da escala $\mathrm{l}(\mathrm{l}=1,2,3$, 4, 5, 6); $\mathrm{NP}_{\mathrm{ij}}=$ efeito da interação volumoso $\times$ concentrado; $\mathrm{NQ}_{\mathrm{il}}=$ efeito da interação volumoso $\times$ escala; $\mathrm{PQ}_{\mathrm{jl}}=$ efeito da interação concentrado $\times$ escala; $\mathrm{NPQ}_{\mathrm{ijl}}=$ efeito da interação volumoso $\times$ concentrado $\times$ escala; e $\mathrm{e}_{\mathrm{ijlr}}=$ erro aleatório associado a cada observação $(r=1,2,3,4,5,6)$.

\section{Resultados e Discussão}

Não houve significância $(\mathrm{P}>0,05)$ para nenhuma das interações testadas. Como demonstrado na Tabela 4, pela análise de variância, não houve efeito significativo $(\mathrm{P}>0,05)$ das escalas de registro do comportamento, o que permite inferir que o comportamento ingestivo de ovinos pode ser avaliado utilizando-se intervalos de observações superiores a cinco minutos.

Esses resultados estão de acordo com os obtidos por Salla et al. (1999), que testaram os tempos de 5, 7, 10 e 15 minutos em comparação ao registro contínuo de observação em quatro vacas Jersey lactantes. Esses autores constataram que o tempo despendido nas atividades foi semelhante em todas as escalas avaliadas, indicando que os animais poderiam ter sido observados a intervalos de até 15 minutos. Todavia, o registro das atividades de alimentação, ruminação e ócio em ovinos pode ser feito a intervalos de até 30 minutos, como ficou constatado neste trabalho.

Esses resultados corroboram os obtidos por Silva et al. (2004), que avaliaram o comportamento ingestivo de novilhas de origem leiteira em pastejo e recomendaram escala de até 30 minutos para avaliação das atividades diárias de alimentação, ruminação e ócio. Na maioria das pesquisas realizadas com o objetivo de determinar o comportamento de animais, em pastejo ou em confinamento, a escolha da escala foi feita de forma totalmente aleatória. Segundo Dutilleul (1997), a adoção de uma escala inadequada pode comprometer os resultados.

Tabela 4 - Tempos despendidos em alimentação, ruminação e ócio ( $\mathrm{min} / \mathrm{dia})$ por ovinos alimentados com dietas contendo capim-elefante amonizado e farelo de cacau (FC) ou torta de dendê (TD)

Table 4 - Time spent eating, ruminating and idle ( $\mathrm{min} /$ day) of sheep fed treated or non-ammoniated treated elephantgrass with or without supplementation of cocoa meal (CM) or palm cake $(P C)$ in the concentrate

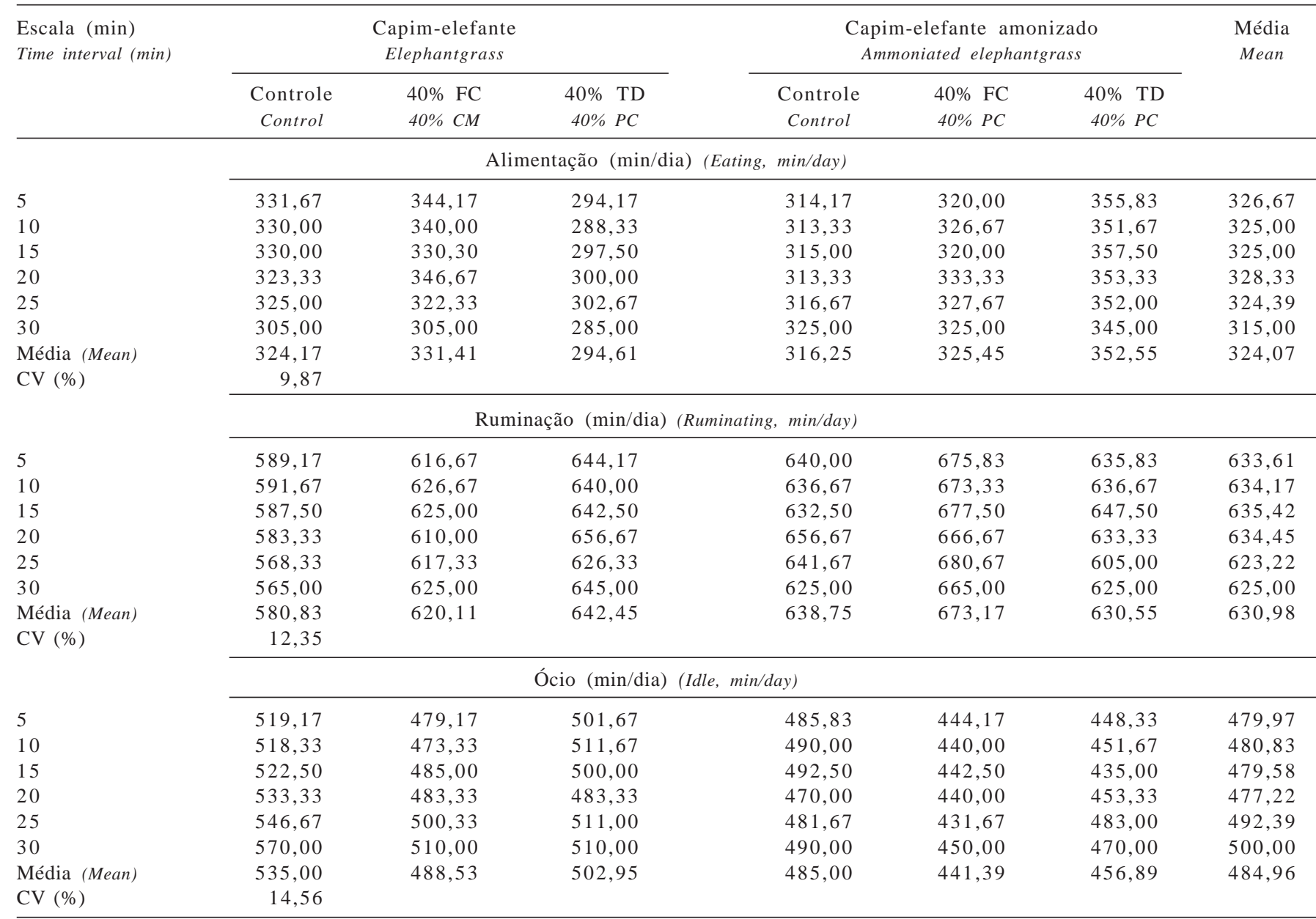


Gary et al. (1970) também não encontraram diferenças entre os tempos de alimentação, ruminação e ócio obtidos pelo registro contínuo das atividades e os obtidos pela escala de 15 minutos. Alguns autores (Salla et al., 1999; Silva et al., 2004), no entanto, observaram diferenças entre os animais, o que pode estar relacionado às características individuais intrínsecas ao comportamento animal ou a outros fatores, como nível de produção, capacidade ingestiva e temperamento. Esse fato não foi observado neste trabalho, provavelmente porque os animais utilizados no experimento apresentavam características bastante homogêneas.

Os dados relacionados ao número de períodos discretos de alimentação, ruminação e ócio (número/dia) e o tempo médio gasto em cada período de atividade são descritos nas Tabelas 5 e 6, respectivamente. As dietas oferecidas não influenciaram o comportamento dos animais $(\mathrm{P}>0,05)$, entretanto, diferenças foram detectadas nas escalas de registros para avaliação do comportamento ingestivo. A escala de cinco minutos foi superior às demais, pois permitiu detectar com maior precisão a freqüência diária despendida para cada atividade. A escolha do intervalo para discretizar as séries temporais, ou seja, o tempo despendido em alimentação, ruminação e descanso, deve ser uma ponderação entre o poder de detectar mudanças na ocorrência das atividades e a precisão, sem, no entanto, incorrer em redundância (Fischer et al., 2000).

Embora muitos pesquisadores (Deswysen et al., 1993; Fischer et al., 1998; Miranda et al., 1999; Portugal et al., 2000; Gonçalves et al., 2001; Costa et al., 2003) tenham optado por escalas superiores a cinco minutos para avaliar o comportamento ingestivo de animais ruminantes, evidenciou-se neste estudo que essa opção só é válida para determinação dos tempos de alimentação, ruminação e ócio. Ao preconizar uma avaliação mais detalhada do comportamento ingestivo, considerando o número de períodos discretos

Tabela 5 - Períodos de alimentação, ruminação e ócio (no/dia) de ovinos alimentados com dietas contendo capim-elefante amonizado e farelo de cacau (FC) ou torta de dendê (TD)

Table 5 - Number of meals, rumination and idle of (n./day) of sheep fed treated or non-ammoniated treated elephantgrass with or without supplementation of cocoa meal (CM) or palm cake (PC) in the concentrate

\begin{tabular}{|c|c|c|c|c|c|c|c|}
\hline \multirow[t]{2}{*}{$\begin{array}{l}\text { Escala (min) } \\
\text { Time interval (min) }\end{array}$} & \multicolumn{3}{|c|}{$\begin{array}{l}\text { Capim-elefante } \\
\text { Elephantgrass }\end{array}$} & \multicolumn{3}{|c|}{$\begin{array}{c}\text { Capim-elefante amonizado } \\
\text { Ammoniated elephantgrass }\end{array}$} & \multirow[t]{2}{*}{$\begin{array}{l}\text { Média } \\
\text { Mean }\end{array}$} \\
\hline & $\begin{array}{c}\text { Controle } \\
\text { Control }\end{array}$ & $\begin{array}{l}40 \% \\
40 \%\end{array}$ & $\begin{array}{c}40 \% \text { TD } \\
40 \% \text { PC }\end{array}$ & $\begin{array}{c}\text { Controle } \\
\text { Control }\end{array}$ & 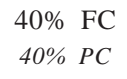 & $\begin{array}{cc}40 \% & \text { TD } \\
40 \% & P C\end{array}$ & \\
\hline & \multicolumn{6}{|c|}{ Período de alimentação (n⿳⺈/dia) (Number of meals, n./day) } & \\
\hline 5 & 11,00 & 15,00 & 9,67 & 12,33 & 11,67 & 11,67 & $11,89 a$ \\
\hline 10 & 7,17 & 9,00 & 8,33 & 9,17 & 10,00 & 8,50 & $8,70 b$ \\
\hline 25 & 4,50 & 5,00 & 5,67 & 6,83 & 7,50 & 4,50 & $5,67 \mathrm{c}$ \\
\hline 30 & 3,33 & 3,50 & 4,17 & 5,33 & 6,33 & 4,50 & $4,53 c$ \\
\hline Média (Mean) & 6,47 & 7,67 & 6,92 & 8,06 & 8,53 & 7,42 & 7,51 \\
\hline \multirow[t]{2}{*}{$\mathrm{CV}(\%)$} & 19,36 & & & & & & \\
\hline & \multicolumn{6}{|c|}{ Período de ruminação ( $\mathrm{n}$ o/dia) (Number of rumination activity, n./day) } & \\
\hline 25 & 10,17 & 10,67 & 13,83 & 10,50 & 11,67 & 10,50 & $11,22 b$ \\
\hline 30 & 9,50 & 9,17 & 11,50 & 9,00 & 10,67 & 8,33 & 9,70c \\
\hline Média (Mean) & 12,78 & 14,11 & 16,75 & 13,56 & 14,67 & 12,81 & 14,11 \\
\hline \multirow[t]{2}{*}{ CV $(\%)$} & 14,58 & & & & & & \\
\hline & \multicolumn{6}{|c|}{ Período de ócio (n-/dia) (Number of idle activity (n./day) } & \\
\hline 5 & 26,33 & 27,17 & 30,67 & 25,33 & 27,83 & 23,33 & $26,78 \mathrm{a}$ \\
\hline 10 & 18,50 & 19,67 & 24,00 & 19,50 & 20,17 & 19,17 & $20,17 b$ \\
\hline 15 & 15,67 & 16,67 & 19,33 & 15,67 & 16,33 & 14,67 & $16,39 b$ \\
\hline 20 & 13,33 & 13,83 & 15,50 & 13,50 & 14,33 & 13,17 & $13,94 \mathrm{c}$ \\
\hline 25 & 12,33 & 11,67 & 15,17 & 11,33 & 11,67 & 9,17 & $11,89 \mathrm{c}$ \\
\hline 30 & 11,17 & 9,67 & 12,00 & 10,83 & 11,50 & 10,17 & $10,89 c$ \\
\hline
\end{tabular}


Tabela 6 - Tempo médio despendido por períodos de alimentação, ruminação e ócio (min/período) em ovinos alimentados com dietas contendo capim-elefante amonizado e farelo de cacau (FC) ou torta de dendê (TD)

Table 6 - Time spent in each meal, rumination and idle activities ( $\mathrm{min} / \mathrm{period}$ ) of sheep fed treated or non-ammoniated treated elephantgrass with or without supplementation of cocoa meal (CM) or palm cake (PC) in the concentrate

\begin{tabular}{|c|c|c|c|c|c|c|c|}
\hline \multirow[t]{2}{*}{$\begin{array}{l}\text { Escala (min) } \\
\text { Time interval (min) }\end{array}$} & \multicolumn{3}{|c|}{$\begin{array}{l}\text { Capim-elefante } \\
\text { Elephantgrass }\end{array}$} & \multicolumn{3}{|c|}{$\begin{array}{l}\text { Capim-elefante amonizado } \\
\text { Ammoniated elephantgrass }\end{array}$} & \multirow[t]{2}{*}{$\begin{array}{l}\text { Média } \\
\text { Mean }\end{array}$} \\
\hline & $\begin{array}{l}\text { Controle } \\
\text { Control }\end{array}$ & $\begin{array}{ll}40 \% & \text { FC } \\
40 \% & C M\end{array}$ & 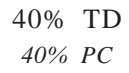 & $\begin{array}{l}\text { Controle } \\
\text { Control }\end{array}$ & 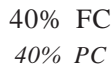 & $\begin{array}{cc}40 \% & \text { TD } \\
40 \% & P C\end{array}$ & \\
\hline
\end{tabular}

\begin{tabular}{|c|c|c|c|c|c|c|c|}
\hline \multirow{2}{*}{5} & \multicolumn{7}{|c|}{ Alimentação (min/período) (Eating, min/period) } \\
\hline & 30,14 & 22,33 & 30,58 & 25,96 & 29,25 & 32,09 & $28,39 c$ \\
\hline 10 & 46,65 & 36,57 & 35,20 & 34,41 & 33,22 & 42,93 & $38,16 c$ \\
\hline 15 & 56,83 & 47,12 & 42,35 & 42,18 & 40,67 & 47,76 & $46,15 b$ \\
\hline 20 & 45,71 & 51,25 & 46,23 & 44,92 & 42,44 & 51,31 & $46,98 \mathrm{~b}$ \\
\hline 25 & 73,14 & 63,44 & 53,90 & 46,35 & 43,65 & 79,47 & $59,99 b$ \\
\hline 30 & 90,83 & 86,74 & 72,08 & 62,55 & 51,03 & 82,80 & $74,34 a$ \\
\hline Média (Mean) & 57,22 & 51,24 & 46,72 & 42,73 & 44,04 & 56,06 & 49,00 \\
\hline \multirow[t]{2}{*}{$\mathrm{CV}(\%)$} & 9,14 & & & & & & \\
\hline & \multicolumn{7}{|c|}{ Ruminação (min/período) (Ruminating, min/period) } \\
\hline 5 & 31,24 & 29,03 & 27,92 & 33,77 & 32,51 & 35,83 & $31,72 \mathrm{c}$ \\
\hline 10 & 41,88 & 36,42 & 32,41 & 39,15 & 43,55 & 41,26 & $39,11 \mathrm{c}$ \\
\hline 15 & 45,19 & 43,62 & 38,88 & 43,74 & 47,63 & 52,37 & $45,24 b$ \\
\hline 20 & 53,46 & 49,87 & 45,35 & 55,68 & 51,45 & 56,44 & $52,04 b$ \\
\hline 25 & 57,40 & 57,88 & 45,39 & 61,32 & 58,18 & 58,11 & $56,38 a$ \\
\hline 30 & 60,18 & 68,01 & 56,96 & 71,95 & 62,36 & 75,58 & $65,84 a$ \\
\hline Média (Mean) & 48,23 & 47,47 & 41,15 & 50,94 & 49,28 & 53,27 & 48,39 \\
\hline \multirow[t]{2}{*}{ CV (\%) } & 7,51 & & & & & & \\
\hline & \multicolumn{7}{|c|}{ Ócio (min/período) (Idle, min/period) } \\
\hline 5 & 19,91 & 17,35 & 16,43 & 19,10 & 15,82 & 20,93 & $18,26 \mathrm{c}$ \\
\hline 10 & 28,26 & 23,69 & 21,38 & 25,06 & 21,51 & 23,81 & $23,95 c$ \\
\hline 15 & 33,49 & 29,09 & 25,96 & 31,46 & 27,12 & 30,11 & $29,54 b$ \\
\hline 20 & 40,29 & 34,74 & 31,14 & 34,68 & 30,17 & 34,55 & $34,26 a$ \\
\hline 25 & 46,12 & 43,13 & 33,72 & 42,31 & 36,46 & 56,51 & $43,04 \mathrm{a}$ \\
\hline 30 & 51,95 & 52,47 & 42,67 & 45,90 & 38,36 & 46,17 & $46,25 a$ \\
\hline Média (Mean) & 36,67 & 33,41 & 28,55 & 33,09 & 28,24 & 35,35 & 32,55 \\
\hline CV (\%) & 11,58 & & & & & & \\
\hline
\end{tabular}

das atividades, a escala de observação de cinco minutos é mais exata e, portanto, mais recomendada, pois permite detectar melhor a freqüência diária de cada atividade, diminuindo as perdas de observações (Tabelas 5 e 6).

Fischer et al. (2000) também afirmaram que a escala ideal para discretizar as séries temporais é a de cinco minutos. Segundo esses autores, esse intervalo resulta em menor perda do número de observações, especialmente para as atividades despendidas com alimentação e ócio.

Como apresentado na Tabela 5, o número de períodos de alimentação e descanso na escala de cinco minutos diferiu dos resultados obtidos nas demais escalas, cujos valores, 11,89 e 26,78, respectivamente, foram superiores aos obtidos nos demais tratamentos. Contudo, a atividade de ruminação determinada na escala de cinco minutos foi superior apenas à observada nas escalas de 15, 20, 25 e 30 minutos, sendo semelhante ao intervalo de 10 minutos. Assim, esses resultados confirmam integralmente os relatados por Fischer et al. (2000).
Avaliando o comportamento ingestivo de cabras lactantes alimentadas com diferentes dietas, Carvalho et al. (2007) buscaram encontrar uma escala ideal para registro do comportamento para esta categoria animal. Esses autores testaram os intervalos de 5, 10, 15 e 20 minutos e recomendaram a escala de 20 minutos, a qual permitiu obter resultados semelhantes aos dos demais tratamentos. Os autores observaram ainda que a discretização das atividades em caprinos pode ser feita no mesmo intervalo de tempo (20 minutos). A escala indicada por Carvalho et al. (2007), de 20 minutos, para o estudo das atividades de alimentação, ruminação e ócio em caprinos poderia ser empregada para ovinos, pois neste estudo ficou evidente que o registro das atividades nestes animais pode ser feito em intervalos de até 30 minutos. Contudo, para a discretização dessas atividades, a escala utilizada pelos autores subestimaria os resultados. Neste estudo com ovinos, ficou evidenciado que a escala de 5 minutos é a mais propícia para discretizar as atividades. 
Resultados semelhantes foram constatados por Silva et al. (2005b), em experimento com novilhas de origem leiteira em confinamento. Esses autores recomendaram a escala de 30 minutos para avaliação das atividades de alimentação, ruminação e ócio e alertaram que, para a discretização das atividades, a escala ideal é a de 5 minutos. Esses resultados permitem inferir que, embora animais ruminantes apresentem hábitos alimentares peculiares, existem fortes indícios de que, dentro de uma mesma escala temporal, as diferentes espécies expressem a mesma tendência para as suas atividades.

\section{Conclusões}

O comportamento ingestivo de ovinos Santa Inês não é alterado pelo consumo de capim-elefante amozinado com $5 \%$ de uréia nem pela adição de $40 \%$ de farelo de cacau ou torta de dendê no concentrado.

As determinações dos tempos de alimentação, ruminação e ócio podem ser feitas utilizando-se intervalos de observações de até 30 minutos.

Para a discretização das séries temporais do comportamento ingestivo, recomenda-se a escala de cinco minutos, pois intervalos superiores provocam subestimação do número de períodos e, portanto, superestimam o tempo médio gasto por período de atividade.

\section{Literatura Citada}

ALBRIGHT, J.L. Feeding behavior of dairy catle. Journal of Dairy Science, v.76, n.2, p.485-498, 1993.

BÜRGER, P.J.; PEREIRA, J.C.; QUEIROZ, A.C. et al. Comportamento ingestivo em bezerros holandeses alimentados com dietas contendo diferentes níveis de concentrado. Revista Brasileira de Zootecnia, v.29, n.1, p.236-242, 2000.

CAMPBELL, C.P.; MARSHALL, S.A.; MANDELL, I.B. et al. Effects of source of dietary neutral detergent fiber on chewing behavior in beef cattle fed pelleted concentrates with or without supplemental roughage. Journal of Animal Science, v.70, n.7, p.894-903, 1992.

CARVALHO, G.G.P.; PIRES, A.J.V.; SILVA, F.F. et al. Comportamento ingestivo de cabras leiteiras alimentadas com farelo de cacau ou torta de dendê. Pesquisa Agropecuária Brasileira, v.39, n.9, p.919-925, 2004.

CARVALHO, G.G.P.; PIRES, A.J.V.; SILVA, H.G.O. et al. Aspectos metodológicos do comportamento ingestivo de cabras lactantes alimentadas com farelo de cacau e torta de dendê. Revista Brasileira de Zootecnia, v.36, n.1, p.103-110, 2007.

COSTA, C.O.; FISCHER, V.; VETROMILLA, M.A.M. et al. Comportamento ingestivo de vacas Jersey confinadas durante a fase inicial da lactação. Revista Brasileira de Zootecnia, v.32, n.2, p.418-424, 2003.

DESWYSEN, A.G.; DUTILleUl, P.A.; GODFRIN, J.P. Nycterohemeral eating and ruminanting patterns in heifers fed grass or corn silage: analysis by finite fourier transform. Journal of Animal Science, v.71, n.10, p.2739-2747, 1993.
DUTILLEUL, P. Incorporating scale in study design: data analysis. In: PETERSON, D.L.; PARKER, V.T. (Eds.) Ecological scale: theory and application. New York: Columbia University Press, 1997. p.1-77.

FISCHER, V.; DESWYSEN, A.G.; DÈSPRES, L. et al. Padrões nectemerais do comportamento ingestivo de ovinos. Revista Brasileira de Zootecnia, v.27, n.2, p.362-369, 1998.

FISCHER, V.; DUTILLEUL, P.; DESWYSEN, A.G. et al. Aplicação de probabilidades de transição de estado dependentes do tempo na análise quantitativa do comportamento ingestivo de ovinos. Parte I. Revista Brasileira de Zootecnia, v.29, n.6, p.18111820, 2000.

GARY, L.A.; SHERRITT, G.W.; HALE, E.B. Behavior of charolais cattle on pasture. Journal of Dairy Science, v.30, n.2, p.303306, 1970.

GONÇALVES, A.L.; LANA, R.P.; RODRIGUES, M.T. et al. Padrão nictemeral do $\mathrm{pH}$ ruminal e comportamento alimentar de cabras leiteiras alimentadas com dietas contendo diferentes relações volumoso:concentrado. Revista Brasileira de Zootecnia, v.30, n.6, p.1886-1892, 2001.

MENDONÇA, S.S.; CAMPOS, J.M.S.; VALADARES FILHO, S.C. et al. Comportamento ingestivo de vacas leiteiras alimentadas com dietas à base de cana-de-açúcar ou silagem de milho. Revista Brasileira de Zootecnia, v.33, n.3, p.723-728, 2004.

MIRANDA, L.F.; QUEIROZ, A.C.; VALADARES FILHO, S.C. et al. Comportamento ingestivo de novilhas leiteiras alimentadas com dietas à base de cana-de-açúcar. Revista Brasileira de Zootecnia, v.28, n.7, p.614-620, 1999.

MOORE, J.A.; POORE, M.H.; SWINGLER, R.S. Influence of roughage source on kinetics of degestion and passage, and on calculated extends of ruminal digestion in beef steers fed $65 \%$ concentrate diets. Journal of Dairy Science, v.68, n.12, p.3412-3420, 1990.

PIRES, A.J.V.; GARCIA, R.; SOUZA, A.L. et al. Avaliação do consumo de silagens de sorgo tratadas com amônia anidra e, ou, sulfeto de sódio na alimentação de novilhas 3/4 Indubrazil/ Holandês. Revista Brasileira de Zootecnia, v.32, n.6, p.1525-1531, 2003.

PORTUGAL, J.A.B.; PIRES, M.F.A.; DURÃES, M.C. Efeito da temperatura ambiente e da umidade relativa do ar sobre a freqüência de ingestão de alimentos e de água e de ruminação em vacas de raça holandesa. Arquivo Brasileiro de Medicina Veterinária e Zootecnia, v.52, n.2, p.154-159, 2000.

QUEIROZ, A.C.; NEVES, J.S.; MIRANDA, L.F. et al. Efeito do nível de fibra e da fonte de proteína sobre o comportamento alimentar de novilhas mestiças holandês-zebu. Arquivo Brasileiro de Medicina Veterinária e Zootecnia, v.53, n.1, p.84-88, 2001.

SALLA, L.E.; FISCHER, V.; FERREIRA, E.X. et al. Comportamento ingestivo de vacas Jersey alimentadas com dietas contendo diferentes fontes de gordura nos primeiros 100 dias de lactação. Revista Brasileira de Zootecnia, v.32, n.3, p.683-689, 2003.

SALLA, L.E.; MORENO, C.B.; FERREIRA, E.X. Avaliação do comportamento de vacas Jersey em lactação - Aspectos Metodológicos I. In: REUNIÃO ANUAL DA SOCIEDADE BRASILEIRA DE ZOOTECNIA, 36., 1999, Porto Alegre. Anais... Porto Alegre: Sociedade Brasileira de Zootecnia, 1999. (CD-ROM).

SILVA, D.J.; QUEIROZ, A.C. Análise de alimentos: métodos químicos e biológicos. 3.ed. Viçosa, MG: UFV, 2002. 235p.

SILVA, R.R.; MAGALHÃES, A.F.; CARVALHO, G.G.P. et al. Comportamento ingestivo de novilhas mestiças de holandês suplementadas em pastejo de Brachiaria. Aspectos metodológicos. Revista Electrócnia de Veterinaria, v.5, n.10, p.1-10, 2004.

SILVA, H.G.O.; PIRES, A.J.V.; SILVA, F.F. et al. Digestibilidade aparente de dietas contendo farelo de cacau ou torta de dendê 
em cabras lactantes. Pesquisa Agropecuária Brasileira, v.40, n.4, p.405-411, 2005a.

SILVA, R.R.; SILVA, F.F.; CARVALHO, G.G.P. et al. Avaliação do comportamento ingestivo de novilhas $3 / 4$ holandês $x$ zebu alimentadas com silagem de capim-elefante acrescida de $10 \%$ de farelo de mandioca. Ciência Animal Brasileira, v.6, n.3, p.134-141, 2005b.
UNIVERSIDADE FEDERAL DE VIÇOSA - UFV. SAEG - Sistema de análises estatísticas e genéticas. Viçosa, MG: 2000. (Apostila).

Recebido: 02/08/06 Aprovado: 26/03/07 